\title{
A Combined Analysis of the CI NQR Transition Frequency and of the Specific Heat in Trichlorofluoromethane *,**
}

\author{
Carlos A. Martín ${ }^{1}$ and Mariano J. Zuriaga ${ }^{2}$ \\ Facultad de Matemática, Astronomía y Física (IMAF), Universidad Nacional de Córdoba, \\ Laprida 854, 5000 Córdoba, República Argentina
}

Z. Naturforsch. 41 a, 412-415 (1986); received July 22, 1985

The temperature dependences of the Nuclear Quadrupole Resonance (NQR) transition frequency $\left(v_{0}\right)$ and of the specific heat $\left(C_{p}\right)$ in Trichlorofluoromethane (TCFM) are analyzed. In the analysis of $v_{\mathrm{O}}$ a procedure is developed and successfully applied that takes advantage of all the transition in the NQR spectrum on a simultaneous basis. An effective value of about $40 \mathrm{~cm}^{-1}$ is determined for the librational frequency of a TCFM molecule, and from the analysis of $C_{p}$ the formation of defects is concluded, which are proposed to be lattice vacancies with a formation energy and entropy of $9.66 \mathrm{~kJ} \cdot \mathrm{mol}^{-1}$ and $29.6 \mathrm{~J} \mathrm{~mol}^{-1} \mathrm{~K}^{-1}$, respectively. The behaviour of $v_{\mathrm{Q}}$ at the higher temperatures and the fade-out of the signals below the melting point are consistent with the presence of lattice vacancies.

\section{Introduction}

This work belongs to one line of research dealing with the effect of lattice vacancies on the NQR parameters. Previous measurements of the specific heat $\left(C_{p}\right.$ vs. $\left.T\right)$ in TCFM [1] show an increasing departure on approaching the melting point from the standard behaviour described by Einstein's or Debye's models, which may be ascribed to vacancy formation. We measured the temperature dependence of $v_{\mathrm{Q}}\left(v_{\mathrm{Q}}\right.$ vs. $\left.T\right)$, for analysing these data according to a model developed in a companion paper [2]. However, we failed in our attempt to obtain quantitative results about vacancies from an analysis of $v_{\mathrm{Q}}$ vs. $T$ since the signals were lost about $20 \mathrm{~K}$ below the melting point, a region where the effect to lattice vacancies is too small to allow for the obtention of satisfactory quantities results. On carrying on the analysis of $v_{\mathrm{Q}}$ vs. $T$ a model is

\footnotetext{
* Presented at the VIIIth International Symposium on Nuclear Quadrupole Resonance Spectroscopy, Darmstadt, July $22-26,1985$.

** This research was partially supported by Consejo Nacional de Investigaciones Científicas y Técnicas (CONICET) and by Consejo de Investigaciones Científicas y Tecnológicas de la Provincia de Córdoba (CONICOR).

1 Fellow of the CONICET. To whom correspondence should be addressed.

2 Holder of an Scholarship granted by CONICET.

Reprint requests to Dr. C. A. Martín, Facultad de Matemática, Astronomía y Física (IMAF), Universidad $\mathrm{Na}$ cional de Córdoba, Laprida 854, 5000 Córdoba, Argentina.
}

proposed which allows to use simultaneously all the lines found in the NQR spectrum.

\section{Experimental}

The sample was purchased from Fluka (Catalog Number 91273) and used as received with no further purification. The signals were detected by means of an SRO type spectrometer, and the transition frequencies were measured by beating the signal with that produced by a synthetizer. The frequencies are measured with an overall error of $\pm 500 \mathrm{~Hz}$ and the temperature to $\pm 0.1 \mathrm{~K}$.

\section{Results and Discussion}

Analysis of $v_{\mathrm{Q}} v s . T$

TFCM exhibits no solid-solid phase transitions [1] and its NQR spectrum shows three transition frequencies, indicating that the three $\mathrm{Cl}$ atoms belonging to the same molecule become chemically inequivalent in passing from the gaseous to the solid state. These unequal splittings are mainly attributed to different lattice contributions to the electric field gradient (EFG) at the $\mathrm{Cl}$ nuclear sites. $v_{\mathrm{Q}}$ vs. $T$ was measured from 85 up to $140 \mathrm{~K}$. The results are shown in Figure 1. The three lines start to broaden at about $120 \mathrm{~K}$, until they fade-out completely at $140 \mathrm{~K}$, that is more than $20 \mathrm{~K}$ below the melting 


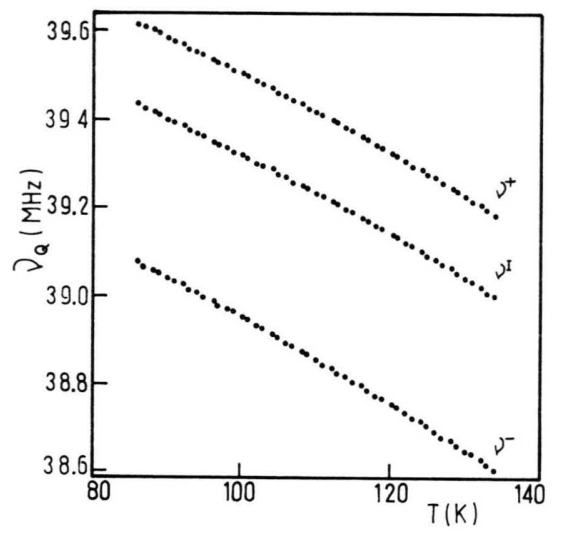

Fig. 1. Temperature dependence of the NQR spectrum.

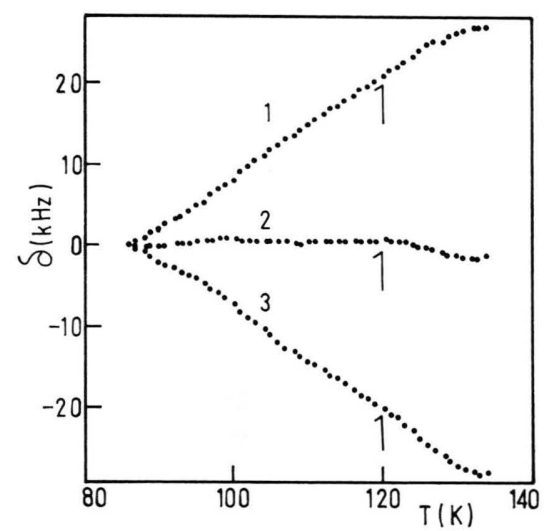

Fig. 2. Frequency difference between pairs of lines, namely $\delta_{i j}=\left[v^{i}(T)-v^{j}(T)\right]-\left[v\left(T_{0}\right)-v^{j}\left(T_{0}\right)\right]$ with $T_{0}=86 \mathrm{~K}$. Curve 1 shows $\delta_{+-}$, curve $2 \delta_{+\mathrm{I}}$ and curve $3\left(-\delta_{\mathrm{I}-}\right)$. These plots clearly show that $v^{+}$vs. $T$ and $v^{\mathrm{I}}$ vs. $T$ are parallel and that $v$ possesses a larger temperature coefficient than $v^{+}$ and $v^{\mathrm{I}}$. The change of behaviour for $T \geqq 120 \mathrm{~K}$ may also be noted.

point which is $162.7 \mathrm{~K}$ [1]. The three lines have large temperature coefficients of approximately $10 \mathrm{kHz} / \mathrm{K}$. Figure 2 depicts the temperature dependence of the difference between pairs of lines; it is clear that some additional mechanism starts to develop at abot $120 \mathrm{~K}$. Therefore we will restrict ourselves to analyse the $v_{\mathrm{Q}}$ vs. $T$ data for $T \leqq 120 \mathrm{~K}$; later on we shall comment on the behaviour for $T \geqq 120 \mathrm{~K}$. write

Following Bayer [3] and Kushida [4] we may

$$
v^{+}=v_{0}\left[1-\frac{3 A}{2 \omega} \operatorname{coth} \frac{\hbar \omega}{2 k T}\right]+v_{\mathrm{c}}^{+},
$$

$$
\begin{aligned}
& v^{\mathrm{I}}=n_{0}\left[1-\frac{3 A}{2 \omega} \operatorname{coth} \frac{\hbar \omega}{2 k T}\right]+v_{\mathrm{c}}^{\mathrm{I}}, \\
& v^{-}=v_{0}\left[1-\frac{3\left(A+A^{\prime}\right)}{2 \omega} \operatorname{coth} \frac{\hbar \omega}{2 k T}\right]+n_{\mathrm{c}}^{-}
\end{aligned}
$$

to describe the temperature dependence of the three lines in such a way as to be consistent with the results shown in Figure 2. $v_{0}$ has been taken as the $v_{\mathrm{Q}}$ corresponding to an isolated molecule; since in this case the three $\mathrm{Cl}$ are equivalent. $v_{\mathrm{c}}^{+}, v_{\mathrm{c}}^{\mathrm{I}}$ and $v_{\mathrm{c}}^{-}$ indicate the lattice contribution to $v_{\mathrm{Q}}$ for each of the three $\mathrm{Cl}$ atoms and represent the mavor contribution to the solid state splittings. The quantities $A$ and $\omega$ are effective values given by

$$
\begin{aligned}
& A=A_{x}+A_{y}+A_{z}, \quad A_{i}=\frac{\hbar}{2 I_{i}}, \\
& \frac{A}{\omega^{2}}=\frac{A_{x}}{\omega_{x}^{2}}+\frac{A_{y}}{\omega_{y}^{2}}+\frac{A_{z}}{\omega_{z}^{2}},
\end{aligned}
$$

where $I_{i}$ and $\omega_{i}(i=x, y, z)$ are the moment of inertia and the librational frequency of the molecule, considered as a rigid body, about the $i$-axis. Using the structural data given in [5] and the axes shown in Fig. $3, A=0.140 \mathrm{~cm}^{-1}$ was found. The EFG asymmetry parameter has been neglected since it is zero in the free molecule and has been found to be very small [6] in similar solid molecular compounds. As may be seen from Figs. 1 and 2, the temperature dependence of $v^{-}$is steeper than that of $v^{+}$and $v^{\mathrm{I}}$; since the normal mode frequencies are the same for the three $\mathrm{Cl}$ atoms, this different behaviour

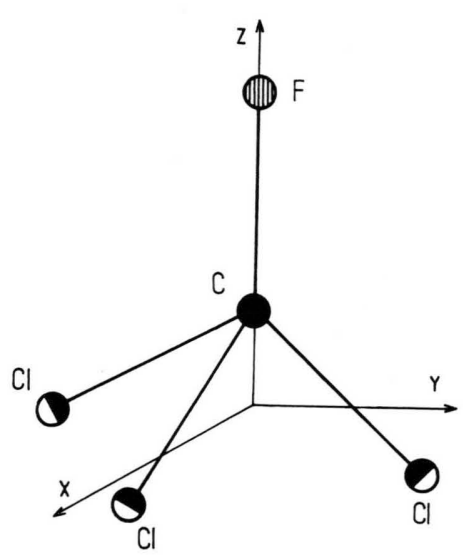

Fig. 3. Schematic diagram of the TCFM molecule. Structural data [5]: $\mathrm{C}-\mathrm{Cl}=1.76 \AA ; \mathrm{C}-\mathrm{F}=1.40 \AA ; \mathrm{Cl}-\mathrm{Cl}=$ $2.91 \AA ; \mathrm{Cl}-\mathrm{F}=2.56 \AA ; \mathrm{Cl}-\mathrm{C}-\mathrm{Cl}=111.5^{\circ}$. 
indicates that the moments of inertia are different, and this is taken into account by $A^{\prime}$ in (3). Additional support to this point is brought by considering $v^{\prime}=\mathrm{d} v / \mathrm{d} T$; the average ratios $v^{\prime 1} / v^{\prime+}=0.9992$ \pm 0.0010 and $v^{\prime-} / v^{\prime+}=1.0679 \pm 0.0014$ are determined by using all the data in the range $85-120 \mathrm{~K}$.

From (1)-(3):

$\left.\begin{array}{l}v^{+}-v^{-} \\ v^{\mathrm{I}}-v^{-}+P_{1} \\ \frac{P_{3}-v^{+}}{P_{2}}\end{array}\right\}=\Delta=v_{\mathrm{c}}^{+}-v_{\mathrm{c}}^{-}+\frac{3 v_{0} A^{\prime}}{2 \omega} \operatorname{coth} \frac{\hbar \omega}{2 k T}$,

where $P_{1}=v^{+}-v^{\mathrm{I}}=v_{\mathrm{c}}^{+}-v_{\mathrm{c}}^{\mathrm{I}}=(187.076 \pm 0.051) \mathrm{kHz}$ is an average value considered to be constant. $P_{2}=A / A^{\prime}=13.958 \pm 0.087$ and $P_{3}=v_{0}+v_{\mathrm{c}}^{+}$ $+A / A^{\prime}\left(v_{\mathrm{c}}^{+}-v_{\mathrm{c}}^{-}\right)=(47.266855 \pm 0.00054) \mathrm{MHz}$ are determined by fitting $P_{2}$ and $P_{3}$ to the equation $v^{+}=-P_{2}\left(v^{+}-v^{-}\right)+P_{3}$.

The $v_{\mathrm{Q}}$ values for various compounds of the series $\mathrm{Cl}_{n} \mathrm{X}_{4-n} \mathrm{C}(x=\mathrm{H}, \mathrm{Cl}, \mathrm{F}$, etc. and $0 \leqq n \leqq 4)$ are all in the neighborhood of $40 \mathrm{MHz}$, indicating that the lattice contribution is not larger than $10 \%$ of $v_{0}$, i.e. $v_{\mathrm{c}} \leqq 4 \mathrm{MHz}$. Therefore $v_{0}$ in (6) may be substituted by $v_{0}+v_{\mathrm{c}}^{+}$without introducing much error (this indeterminacy in $v_{0}$ will produce an error in $\omega$ of at most $5 \%$ ). Neglecting $v_{c}^{+}$in (1) it is found that $v_{0}=(39.756 \pm 0.005) \mathrm{MHz}$. Substituting this value into (6) and using an $\omega$ temperature dependence as that proposed by Brown (7), i.e. $\omega=\omega_{0}(1-C T)$,

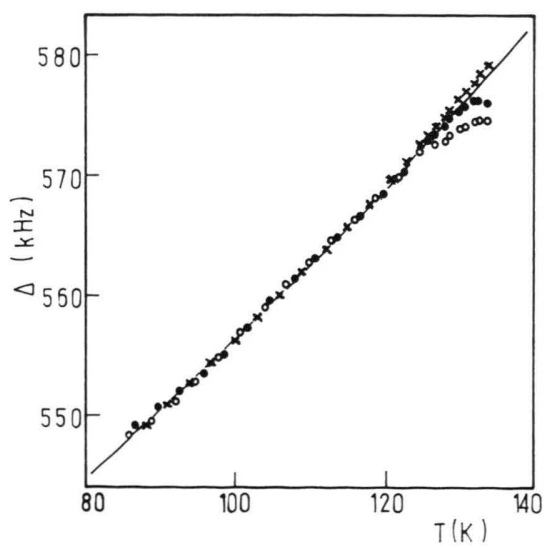

Fig. 4. The solid line corresponds to the plot of (6) with the parameters given in the text. Open circles correspond to $v^{+}(T)-v^{-}(T)$; solid circles to $v^{\mathrm{I}}(T)-v(T)+P_{1}$; and crosses to $\left(P_{3}-v+(T)\right) / P_{2}$. the following value are obtained:

$$
\begin{aligned}
& v_{\mathrm{c}}^{+}-v_{\mathrm{c}}^{-}=(502.2 \pm 1.9) \mathrm{kMz} ; \\
& \omega_{0}=(43.4 \pm 1.6) \mathrm{cm}^{-1}, \quad \text { and } \\
& C=(8.6 \pm 1.7) \cdot 10^{-4} \mathrm{~K}^{-1} .
\end{aligned}
$$

Unfortunately there are no values, obtained by means of other techniques, to compare with. However, the values of $\omega_{0}$ and $C$ may be considered very reasonable when compared to those measured for chloroform (8). These values are also supported by the good description they provide for all of the data, as shown in Figure 4. The appearance of an additional mechanism, becoming efficient above $120 \mathrm{~K}$ and increasing the temperature dependence of $v_{\mathrm{Q}}$, is clearly seen.

Analysis of $C_{p}$ vs. $T$

Since there are no data about the crystal structure it will be assumed that there is only one molecule per unit cell. This gives rise to 15 normal mode frequencies at the Brillouin zone center: 3 acoustic,

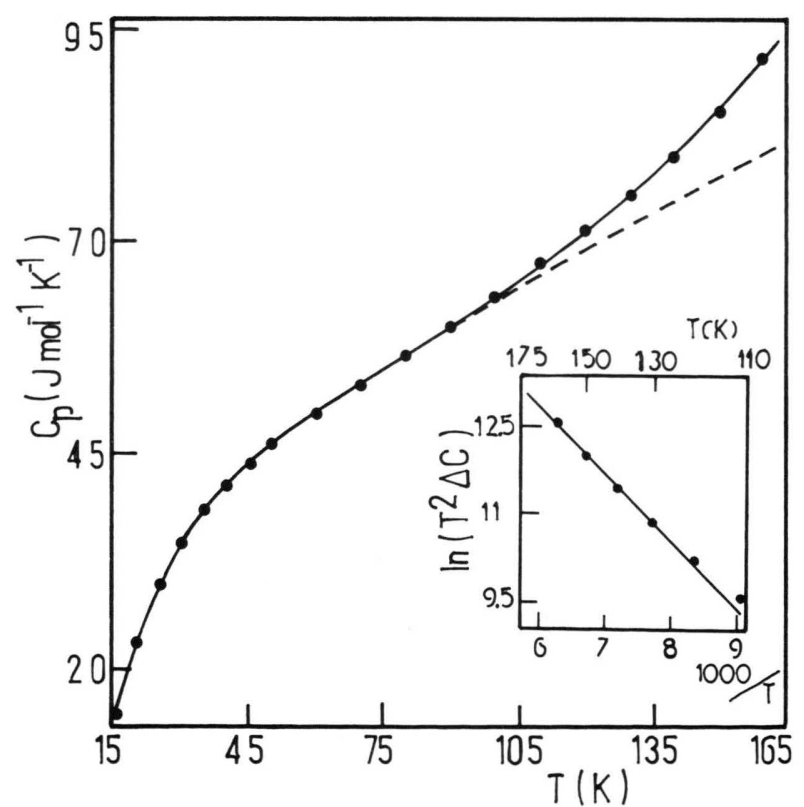

Fig. 5. Solid circles correspond to $C_{p}$ vs. $T$ data [1]. The solid line is obtained employing (7) with the parameters given in text. Dashed line is obtained from (7) but not including defect formation. The insert shows $\ln \left(T^{2} \Delta C\right)$ $\left(T^{2} \Delta C\right.$ in $\left.\mathrm{J} \mathrm{mol}^{-1} \mathrm{~K}\right)$ as a function of $100 / T$ ( $T$ in $\left.\mathrm{K}\right)$. $\triangle C$ is the difference between the experimental data (solid circles) and the dashed line. 
3 librational optic and 9 internal optic ones. The frequencies of the 9 internal modes have been determined [9] to be: $v_{1}=1085 \mathrm{~cm}^{-1} ; v_{2}=v_{3}=846$ $\mathrm{cm}^{-1} ; \quad v_{4}=534 \mathrm{~cm}^{-1} ; \quad v_{5}=v_{6}=400 \mathrm{~cm}^{-1} ; \quad v_{7}=351$ $\mathrm{cm}^{-1}$ and $v_{8}=v_{9}=245 \mathrm{~cm}^{-1}$ and are assumed to be temperature independent. The three optic librational modes are taken as those produced by the above analysis of $v_{\mathrm{Q}}$ vs. $T$, i.e. $v_{10}=v_{11}=v_{12}=43.4$ $\left(1-8.6 \cdot 10^{-4} T\right) \mathrm{cm}^{-1}$. For the three translational acoustic modes we assume a Debye frequency of the form $v_{13}=v_{14}=v_{15}=v_{0 \mathrm{D}}\left(1-C_{\mathrm{D}} T\right)$ in a similar form to that of the librational modes. Following Wallace [10] it can be shown that

$$
\begin{gathered}
C_{p}=R\left[\sum_{j=1}^{12} \frac{1}{1 \mid C_{j} T} \frac{x_{j} e^{x_{j}}}{\left(e^{x} j-1\right)^{2}}+\frac{9}{x_{\mathrm{D}}^{3}} \frac{1}{1-C_{\mathrm{D}} T}\right. \\
\left.\cdot \int_{0}^{x_{\mathrm{D}}} \frac{x^{4} e^{x}}{\left(e^{x}-1\right)^{2}} \mathrm{~d} x+\left(\frac{\varepsilon_{\mathrm{f}}}{k T}\right)^{2} \exp \left[\left(s_{\mathrm{f}}-\varepsilon_{\mathrm{f}} / T\right) / k\right]\right],
\end{gathered}
$$

where $x_{j}=h v_{j} / k T, s_{\mathrm{f}}$ and $\varepsilon_{\mathrm{f}}$ are the entropy and energy associated with the creation of a vacancy, and $R$ is the gas constant. The first term takes into

[1] D. W. Osborne, C. S. Garner, R. N. Doescher, and D. M. Yost, J. Amer. Chem. Soc. 63, 3496 (1941).

[2] M. J. Zuriaga and C. A. Martín, companion paper in this issue.

[3] H. Bayer, Z. Phys. 130, 227 (1951).

[4] T. Kushida, J. Sci. Hiroshima Univ. A 19, 327 (1955).

[5] L. O. Brockway, J. Phys. Chem. 41, 185 and 747 (1937).

[6] G. Litzistorf, S. Sengupta, and E. A. C. Lucken, J. Magn. Reson. 42, 307 (1981). account the contribution of the optic modes, the second the contribution of the acoustic modes, and the third accounts for formation of defects, which in the present case are assigned to vacancies [11]. The resulting values of the fit are $v_{0 \mathrm{D}}=(72.5 \pm 1.0)$ $\mathrm{cm}^{-1} ; C=(5.95 \pm 0.88) \cdot 10^{-4} \mathrm{~K}^{-1} ; S_{\mathrm{f}}=(29.6 \pm 3.0)$ $\mathrm{J} \mathrm{mol}^{-1} \mathrm{~K}^{-1} ; \quad \varepsilon_{\mathrm{f}}=(9.66 \pm 0.69) \mathrm{kJ} \mathrm{mol}^{-1}$. A comparison with $C_{p}$ vs. $T$ data is shown in Figure 5. The insert clearly shows that we are in fact dealing with defect creating. The values determined for the various quantities are very reasonable [11].

A method was developed which allows to analyze $v_{\mathrm{Q}}$ vs. $T$, for the various lines in the same compound in a simultaneous form. The analysis of $C_{p}$ vs. $T$ is consistent wih the presence of vacancies, and this is qualitatively confirmed by the analysis of $v_{\mathrm{Q}}$ vs. $T$, as may be seen in Figs. 2 and 4. This indicates that vacancies move throughout the crystal very slowly as compare to any of the $v_{\mathrm{c}}$ [2]. Further analysis will be possible as soon as $v_{\mathrm{Q}}$ can be measured at higher temperatures, and also when line shape and relaxation data become available.

7] R. J. C. Brown, J. Chem. Phys. 32, 116 (1960).

[8] B. Andrews, A. Anderson, and B. Torrie, Chem. Phys. Lett. 104, 65 (1984).

[9] R. B. Bernstein, J. P. Zietlow, and F. F. Cleveland, J. Chem. Phys. 21, 1778 (1953).

[10] D. C. Wallace, Thermodynamics of Crystals, Wiley, New York 1972.

[11] A. Seeger, D. Schumacher, W. Schilling, and J. Diehl, Vacancies and Intersticials in Metals, North-Holland, Amsterdam 1970. 